\title{
Evaluation of Probabilistic Transformations for Evidential Data Association
}

\author{
Mohammed Boumediene ${ }^{1,2(\bowtie)}(\mathbb{0})$ and Jean Dezert ${ }^{3}(\mathbb{C}$
}

1 Signals and Images Laboratory, USTO, Mohamed Boudiaf, Oran, Algeria

2 National Institute of Telecommunications and ICT, Abdelhafid Boussouf, Oran, Algeria

mboumediene@inttic.dz

3 The French Aerospace Lab, ONERA - DTIS, 92320 Palaiseau, France

jean.dezert@onera.fr

\begin{abstract}
Data association is one of the main tasks to achieve in perception applications. Its aim is to match the sensor detections to the known objects. To treat such issue, recent research focus on the evidential approach using belief functions, which are interpreted as an extension of the probabilistic model for reasoning about uncertainty. The data fusion process begins by quantifying sensor data by belief masses. Thereafter, these masses are combined in order to provide more accurate information. Finally, a probabilistic approximation of these combined masses is done to make-decision on associations. Several probabilistic transformations have been proposed in the literature. However, to the best of our knowledge, these transformations have been evaluated only on simulated examples. For this reason, the objective of this paper is to benchmark most of interesting probabilistic transformations on real-data in order to evaluate their performances for the autonomous vehicle perception problematic.
\end{abstract}

Keywords: Data association - Evidential theory $\cdot$ Belief functions Probabilistic transformation

\section{Introduction}

Multiple Target Tracking (MTT) is important in perception applications (autonomous vehicle, surveillance, etc.). The MTT system is usually based on two main steps: data association and tracking. The first step associates detected objects in the perceived scene, called targets, to known objects characterized by their predicted tracks. The second step estimates the track states over time typically thanks to Kalman Filters [1], or improved state estimation techniques (like particle filters, etc). Nevertheless, bad associations provide wrong track estimation and then leads to false perception results. 
The data association problem is usually resolved by Bayesian theory $[1,2]$. Several methods have been proposed as the Global Nearest Neighbor (GNN) method, the Probabilistic Data Association Filter (PDAF), and the Multiple Hypothesis Tracking (MHT) [3,12,22]. However, the Bayesian theory doesn't manage efficiently data imperfection due to the lack of knowledge we can have on sensor quality, reliability, etc. To circumvent this drawback, the Evidential theory $[9,25]$ appears as an interesting approach because of its ability to model and deal with epistemic uncertainty. Its provides a theoretical framework to manage ignorance and data imperfection.

Several evidential data association approaches have been proposed $[6,10,20$, $23]$ in the framework of belief functions. Rombaut [23] uses the Evidential theory to measure the confidence of the association between perceived and known obstacles. To manage efficiently objects appearance and disappearance, Gruyer and Cherfaoui [15] propose the bi-directional data association. The first direction concerns the target-to-track pairings which provides a good way to manage the appearance of the new tracks. The second direction concerns the track-totarget pairings and then manage disappearance of tracks. This approach has been extended by Mercier et al. [20] to track vehicles by using a global optimization to make assignment decisions. To reduce the complexity for real-time applications, a local optimization has been used $[5,6]$. For all these methods, the data fusion process begins by defining belief masses from sensor information and prior knowledge. These masses represent the belief and ignorance on the assignment hypotheses. Thereafter, the masses are combined in order to provide a complete information of the considered problem. Finally, to make a decision, the belief masses are classically approximated by a probability measure thanks to a chosen probabilistic transformation.

For data association applications, the widely used probabilistic transformation (i.e. approximation) is the pignistic transformation $[5,6,17,20]$. This transformation is based on a simple mapping process from belief to probability domain. However, several published works criticize the pignistic transformation and propose generalized and/or alternative transformations $[7,8,11,19,21,30]$. To our knowledge, the proposed transformations have been evaluated by their authors only on simulated examples. The main objective of this paper is to compare these transformations on real-data in order to determine which one is well-suited for assignment problems.

The rest of the paper is structured as follows. Section 2 recalls the basics of belief functions and their uses in data association problems. In Sect.3, the most appealing probabilistic transformations are presented and compared on the well-known KITTI public database in Sect. 4 . Finally, Sect. 5 concludes the paper. 


\section{Belief Functions for Data Association}

To select "best" associations, the data fusion process consists in four steps: modeling, estimation, combination and decision-making. This section presents their definitions and principles.

\subsection{Basic Fundamentals}

The Belief Functions (BF) have been introduced by Shafer [25] based on Dempster's researches [9]. They offer a theoretical framework for reasoning about uncertainty. Let's consider a problem where we have an exhaustive list of hypotheses $\left(H_{j}\right)$ which are mutually exclusive. They define a so-called frame of discernment $\Theta$ :

$$
\Theta=\bigcup_{j=1}^{k}\left\{H_{j}\right\} \text { with } H_{i} \cap H_{j}=\emptyset
$$

The power set $2^{\Theta}$ is the set of all subsets of $\Theta$, that is:

$$
2^{\Theta}=\left\{\emptyset, H_{1}, \ldots, H_{k}, \ldots,\left\{H_{1}, H_{2}, H_{3}\right\}, \ldots, \Theta\right\}
$$

The proposition $A=\left\{H_{1}, H_{2}, H_{3}\right\}$ represents the disjunction meaning that either $H_{1}$ or $H_{2}$ or $H_{3}$ can be the solution to the problem under concern. In other words, $A$ represents a partial ignorance if $A$ is the disjunction of several elements of $\Theta$. The union of all hypotheses $\Theta$ represents the total ignorance and $\emptyset$ is the empty set that represents the impossible solution (interpreted usually as the conflicting information).

The truthfulness of each proposition $A \in 2^{\Theta}$ issued from source $j$ is modeled by a basic belief assignment (bba) $m_{j}^{\Theta}(A)$ :

$$
m_{j}^{\Theta}: 2^{\Theta} \rightarrow[0,1], \sum_{A \in 2^{\Theta}} m_{j}^{\Theta}(A)=1
$$

Thereafter, the different bbas $\left(m_{j}^{\Theta}\right)$ are combined which provides a global knowledge of the considered problem. Several rules of combination have been proposed [29], the conjunctive operator is widely used in many rules proposed in the literature for the combination of sources of evidence. For instance, Shafer [25] did propose Dempster's rule of combination below which is nothing but the normalized version of the conjunctive rule [26]:

$$
\left\{\begin{array}{l}
m_{D S}^{\Theta}(A)=\frac{1}{1-K} \sum_{A_{1} \cap \ldots \cap A_{p}=A} \prod_{j=1}^{p} m_{j}^{\Theta}\left(A_{j}\right) \\
m_{D S}^{\Theta}(\emptyset)=0
\end{array}\right.
$$

where $K$ is a normalized coefficient:

$$
K=\sum_{A_{1} \cap \ldots \cap A_{p}=\emptyset} \prod_{j=1}^{p} m_{j}^{\Theta}\left(A_{j}\right) .
$$


Finally, in order to make decisions in $\Theta$, a probabilistic approximation of the combined bbas $\left(m_{D S}^{\Theta}(A)\right)$ is usually done. The upper and the lower bounds of the unknown probability $P(A)$ are defined by the belief $\operatorname{Bel}(A)$ and the plausibility $\mathrm{Pl}(A)$ functions given respectively by:

$$
\left\{\begin{array}{l}
\operatorname{Bel}(A)=\sum_{B \subseteq A} m_{D S}^{\Theta}(B) \\
P l(A)=\sum_{B \cap A \neq \emptyset} m_{D S}^{\Theta}(B)
\end{array}\right.
$$

\subsection{Belief Modeling}

The data association problem can be analyzed from two points of view: target-totrack and track-to-target association. Consequently, two frames of discernment are defined: $\Theta_{i, .}$ and $\Theta_{., j}, i=1, \ldots, n$, with $n$ the number of targets, and $j=$ $1, \ldots, m$, with $m$ the number of tracks:

$$
\begin{aligned}
& \Theta_{i, .}=\left\{Y_{(i, 1)}, Y_{(i, 2)}, \ldots, Y_{(i, m)}, Y_{(i, *)}\right\} \\
& \Theta_{., j}=\left\{X_{(1, j)}, X_{(2, j)}, \ldots, X_{(n, j)}, X_{(*, j)}\right\}
\end{aligned}
$$

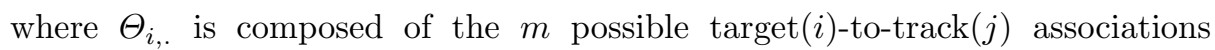
denoted $Y_{(i, j)}$. The hypothesis of appearance is represented by $Y_{(i, *)}{ }^{1} \cdot \Theta_{., j}$ contains the $n$ possible $\operatorname{track}(j)$-to-target $(i)$ associations denoted $X_{(i, j)}$, and $X_{(*, j)}$ is the track disappearance.

\subsection{Basic Belief Assignment}

For target-to-track assignment, three bba's are used to answer the question "Is target $X_{i}$ associated with track $Y_{j}$ ?":

- $m_{j}^{\Theta_{i, \cdot}}\left(Y_{(i, j)}\right)$ : belief in " $X_{i}$ is associated with $Y_{j}$ ",

- $m_{j}^{\Theta_{i, .}}\left(\overline{Y_{(i, j)}}\right)$ : belief in " $X_{i}$ is not associated with $Y_{j}$ ",

- $m_{j}^{\Theta_{i, .}}\left(\Theta_{i, .}\right)$ : the degree of ignorance.

The recent benchmark [4] on huge real data shows that the most suited model is the non-antagonist model $[14,23]$ which is defined as follows:

$$
\begin{aligned}
& m_{j}^{\Theta_{i, .}}\left(Y_{(i, j)}\right)= \begin{cases}0 & , I_{i, j} \in[0, \tau] \\
\Phi_{1}\left(I_{i, j}\right) & , I_{i, j} \in[\tau, 1]\end{cases} \\
& m_{j}^{\Theta_{i, .}}\left(\overline{Y_{(i, j)}}\right)= \begin{cases}\Phi_{2}\left(I_{i, j}\right) & , I_{i, j} \in[0, \tau] \\
0 & , I_{i, j} \in[\tau, 1]\end{cases}
\end{aligned}
$$

\footnotetext{
${ }^{1} Y_{(i, *)}$ refers to the fact that no track is assigned to the $\operatorname{target}(i)$.

${ }^{2} \overline{Y_{(i, j)}}$ defines the complementary hypothesis of $Y_{(i, j)}$, $\overline{Y_{(i, j)}}=\left\{Y_{(i, 1)}, \ldots, Y_{(i, j-1)}, Y_{(i, j+1)}, \ldots, Y_{(i, m)}, Y_{(i, *)}\right\}$.
} 


$$
m_{j}^{\Theta_{i, \cdot}}\left(\Theta_{i, .}\right)=1-m_{j}^{\Theta_{i, .}}\left(Y_{(i, j)}\right)-m_{j}^{\Theta_{i, \cdot}}\left(\overline{Y_{(i, j)}}\right)
$$

where $0<\tau<1$ represents the impartiality of the association process and $I_{i, j} \in[0,1]$ is an index of similarity between $X_{i}$ and $Y_{j} . \Phi_{1}($.$) and \Phi_{2}($.$) are two$ cosine functions defined by:

$$
\left\{\begin{array}{l}
\Phi_{1}\left(I_{i, j}\right)=\frac{\alpha}{2}\left[1-\cos \left(\pi \frac{I_{i, j}-\tau}{\tau}\right)\right] \\
\Phi_{2}\left(I_{i, j}\right)=\frac{\alpha}{2}\left[1+\cos \left(\pi \frac{I_{i, j}}{\tau}\right)\right],
\end{array}\right.
$$

where $0<\alpha<1$ is the reliability factor of the data source. In the same manner, belief masses are generated for the track-to-target assignment.

\subsection{Belief Combination}

Based on Dempster's rule (4), the combined masses $m^{\Theta_{i, .}}\left(\right.$ and $m^{\Theta_{., j}}$ ) over $2^{\Theta_{i, \text {. }}}$ (and $2^{\Theta, j}$ ) can be computed as follows [24]:

$$
\begin{aligned}
& m^{\Theta_{i, .}}\left(Y_{(i, j)}\right)=K \cdot m_{j}^{\Theta_{i, .}}\left(Y_{(i, j)}\right) \prod_{\substack{a=1 \\
a \neq j}}^{m} \alpha_{(i, a)} \\
& m^{\Theta_{i, .}}\left(\left\{Y_{(i, j)}, \ldots, Y_{(i, l)}, Y_{(i, *)}\right\}\right)=K \cdot \gamma_{(i,(j, \ldots, l))} \prod^{m} \beta_{(i, a)} \\
& a=1 \\
& a \neq j \\
& \text {...... } \\
& m^{\Theta_{i, .}}\left(Y_{(i, *)}\right)=K \cdot \prod_{a=1}^{m} \beta_{(i, a)} \\
& m^{\Theta_{i, .}}\left(\Theta_{i, .}\right)=K \cdot \prod_{a=1}^{m} m_{a}^{\Theta_{i, .}}\left(\Theta_{i, .}\right)
\end{aligned}
$$

with:

$$
\left\{\begin{array}{l}
\alpha_{(i, a)}=1-m_{a}^{\Theta_{i, .}}\left(Y_{(i, a)}\right) \\
\beta_{(i, a)}=m_{a}^{\Theta_{i, .}}\left(\overline{Y_{(i, a)}}\right) \\
\gamma_{(i,(j, \ldots, l))}=m_{j}^{\Theta_{i, .}}\left(\Theta_{i, .}\right) \ldots m_{l}^{\Theta_{i, .}}\left(\Theta_{i, .}\right) \\
K=\left[\prod_{a=1}^{m} \alpha_{(i, a)}+\sum_{a=1}^{m} m_{a}^{\Theta_{i, .}}\left(Y_{(i, a)}\right) \prod_{\substack{b=1 \\
b \neq a}}^{m} \alpha_{(i, b)}\right]^{-1}
\end{array}\right.
$$

\subsection{Decision-Making}

Finally, the probabilities matrix $P_{i, .}\left(P_{., j}\right)$ is obtained by using a probabilistic transformation. Table 1 presents the $P_{i, .}$ matrix where each line defines the association probabilities of the target $X_{i}$ with all tracks $Y_{j} . P_{i, .}\left(Y_{(i, *)}\right)$ represents the appearance probability of $X_{i}$. 
Table 1. Probabilities of target-to-track associations

\begin{tabular}{l|l|l|l|l}
\hline$P_{i, .}()$. & $Y_{1}$ & $\ldots$ & $Y_{m}$ & $Y_{*}$ \\
\hline$X_{1}$ & $P_{1, .}\left(Y_{(1,1)}\right)$ & $\ldots$ & $P_{1, .}\left(Y_{(1, m)}\right)$ & $P_{1, .}\left(Y_{(1, *)}\right)$ \\
\hline$X_{2}$ & $P_{2, .}\left(Y_{(2,1)}\right)$ & $\ldots$ & $P_{2, .}\left(Y_{(2, m)}\right)$ & $P_{2, .}\left(Y_{(2, *)}\right)$ \\
\hline$\vdots$ & $\vdots$ & $\vdots$ & $\vdots$ & $\vdots$ \\
\hline$X_{n}$ & $P_{n, .}\left(Y_{(n, 1)}\right)$ & $\ldots$ & $P_{n, .}\left(Y_{(n, m)}\right)$ & $P_{n, .}\left(Y_{(n, *)}\right)$ \\
\hline
\end{tabular}

The association decisions are made by using a global or a local optimization strategy. The Joint Pignistic Probability (JPP) [20] selects associations that maximize the probability product. However, this global optimization is timeconsuming and can select doubtful local associations. To cope these drawbacks, local optimizations have been proposed as the Local Pignistic Probability (LPP). Interested readers in the benchmark of these algorithms can refer to $[17,18]$.

\section{Probabilistic Transformations}

The generalized formula of the probabilistic transformation can be defined as follows:

$$
P_{i, .}\left(Y_{(i, j)}\right)=m^{\Theta_{i, .}}\left(Y_{(i, j)}\right)+\sum_{\substack{A \in 2^{\Theta_{i, .}} \\ Y_{(i, j)} \subset A}} T\left(Y_{(i, j)}, A\right) \cdot m^{\Theta_{i, .}}(A)
$$

where $A$ represents the partial/global ignorance about the association of target $X_{i}$ and $T\left(Y_{(i, j)}, A\right)$ represents the rate of the ignorance mass $m^{\Theta_{i, .}}(A)$ which is transferred to singleton $Y_{(i, j)}$.

Several probabilistic transformations have been proposed in the literature. In this section, only the most interesting ones are presented.

\subsection{Pignistic Probability}

The pignistic transformation denoted by BetP and proposed by Smets $[27,28]$ is still widely used for evidential data association applications $[6,14,16,20]$. This transformation redistributes equitably the mass of ignorance on singletons as follows:

$$
T_{B e t P_{i, .}}\left(Y_{(i, j)}, A\right)=\frac{1}{|A|}
$$


where $|A|$ represents the cardinality of the subset A. However, the pignistic transformation (14) ignores the bbas of singletons which can be considered as a crude commitment. BetP is easy to implement because it has a low complexity due to its simple redistribution process.

\subsection{Dezert-Smarandache Probability}

Besides of the cardinality, Dezert-Smarandache Probability (DSmP) transformation [11] considers the values of masses when transferring ignorance on singletons:

$$
T_{D S m P_{i, .}}\left(Y_{(i, j)}, A\right)=\frac{m^{\Theta_{i, .}\left(Y_{(i, j)}\right)+\epsilon}}{\sum_{Y_{(i, k)} \subset A} m^{\Theta_{i, .}}\left(Y_{(i, k)}\right)+\epsilon \cdot|A|}
$$

The value of the tuning parameter $\epsilon \geq 0$ is used to adjust the effect of focal element's cardinality in the proportional redistribution, and to make $D S m P$ defined and computable when encountering zero masses. Typically, one takes $\epsilon=0.001$. The smaller $\epsilon$, the better approximation of probability measure we get [11]. DSmP allows to obtain in general a higher Probabilistic Information Content (PIC) [31] than BetP because it uses more information than BetP for its establishment. The PIC indicates the level of the available knowledge to make a correct decision. $P I C=0$ indicates that no knowledge exists to take a correct decision.

\subsection{MultiScale Probability}

The Multiscale Probability (MulP) transformation [19] highlights the proportion of each hypothesis in the frame of discernment by using a difference function between belief and plausibility:

$$
T_{M u l P_{i, .}}\left(Y_{(i, j)}, A\right)=\frac{\left(P l^{\Theta_{i, .}}\left(Y_{(i, j)}\right)-B e l^{\Theta_{i, .}}\left(Y_{(i, j)}\right)\right)^{q}}{\sum_{Y_{(i, k)} \subset A}\left(P l^{\Theta_{i, .}}\left(Y_{(i, k)}\right)-B e l^{\Theta_{i, .}}\left(Y_{(i, k)}\right)\right)^{q}},
$$

where $q \geq 0$ is a factor used to amend the proportion of the difference $(P l(\cdot)-$ $\operatorname{Bel}(\cdot))$. However, the $T_{M u l P_{i,}}$ is not defined $\left(\frac{0}{0}\right)$ when $m(\cdot)$ is a Bayesian mass $(P l(\cdot)=\operatorname{Bel}(\cdot))$. 


\subsection{Sudano's Probabilities}

Sudano proposes several alternatives to BetP as the Proportional Plausibility $(\mathrm{PrPl})$ and the Proportional Belief $(\mathrm{PrBel})$ transformations $[11,30]$. Those latter redistribute respectively the ignorance mass according to the normalized plausibility and belief functions:

$$
\begin{aligned}
T_{P r P l_{i, .}}\left(Y_{(i, j)}, A\right) & =\frac{P l^{\Theta_{i, .}}\left(Y_{(i, j)}\right)}{\sum_{Y_{(i, k)} \subset A} P l^{\Theta_{i, .}}\left(Y_{(i, k)}\right)} \\
T_{P r B e l_{i, .}}\left(Y_{(i, j)}, A\right) & =\frac{B e l^{\Theta_{i, .}\left(Y_{(i, j)}\right)}}{\sum_{Y_{(i, k)} \subset A} B e l^{\Theta_{i, .}}\left(Y_{(i, k)}\right)}
\end{aligned}
$$

\subsection{Pan's Probabilities}

Other proportional transformations have been proposed in [21]. Those transformations assume that the $b b a$ are proportional to a function $S(\cdot)$ which is based on the belief and the plausibility:

$$
T_{P r B P_{i, .}}\left(Y_{(i, j)}, A\right)=\frac{S(i, j)}{\sum_{Y_{(i, k)} \subset A} S(i, k)},
$$

where different definitions of $S$ have been proposed:

$$
\left\{\begin{array}{l}
\operatorname{PrBP} 1_{i, .}: S(i, j)=P l^{\Theta_{i, .}}\left(Y_{(i, j)}\right) \cdot \operatorname{Bel}^{\Theta_{i, .}\left(Y_{(i, j)}\right)} \\
\operatorname{PrBP} 2_{i, .}: S(i, j)=B e l^{\Theta_{i, .}}\left(Y_{(i, j)}\right) \cdot\left(1-P l^{\Theta_{i, .}}\left(Y_{(i, j)}\right)\right)^{-1} \\
\operatorname{PrBP} 3_{i, .}: S(i, j)=P l^{\Theta_{i, .}}\left(Y_{(i, j)}\right) \cdot\left(1-\operatorname{Bel}^{\Theta_{i, .}}\left(Y_{(i, j)}\right)\right)^{-1}
\end{array}\right.
$$

\section{Results}

This section presents a benchmark of the probabilistic transformations in the framework of the object association system for autonomous vehicles. The aim is to assign detected objects in the scene (targets) to known ones (tracks). The transformations have been evaluated on real data. 

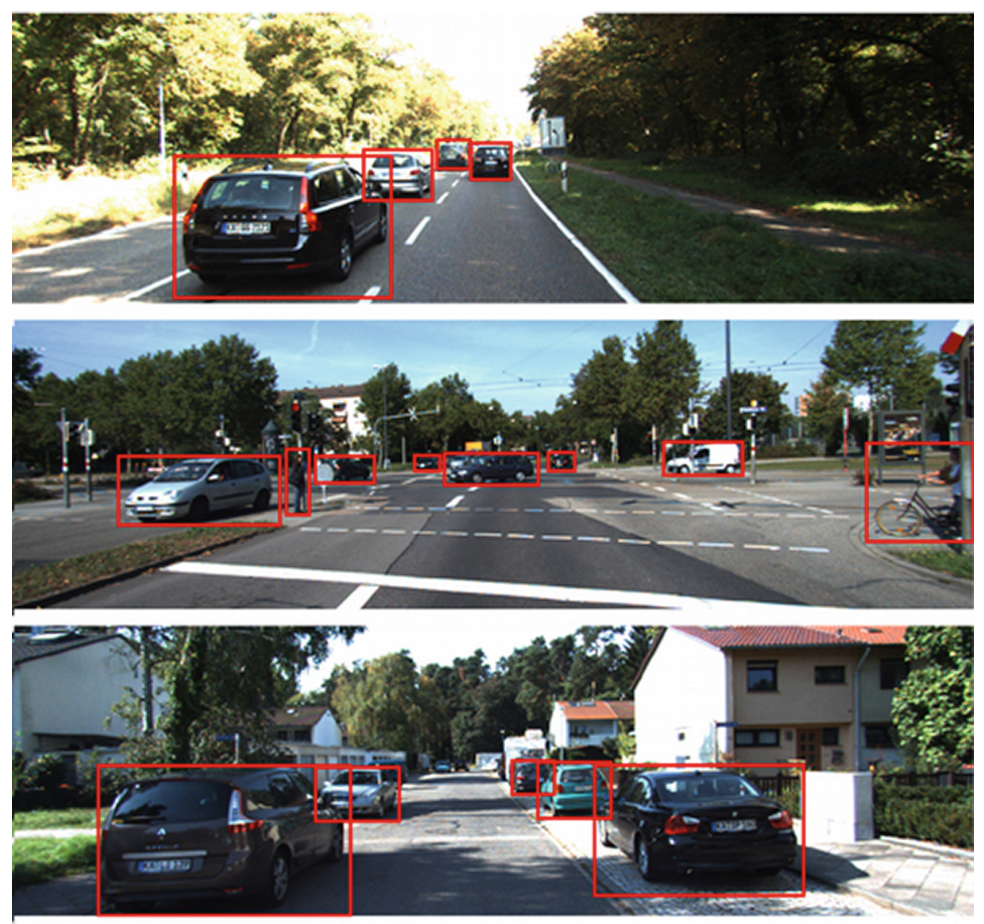

Fig. 1. Examples of images provided by KITTI [4].

The KITTI dataset ${ }^{3}$ provides 21 sequences recorded from cameras mounted on a moving vehicle on urban roads [13]. To our knowledge, no comparison of probabilistic transformations has been done on real data where more than 30000 associations have been observed. These latter cover different road scenario as shown in Fig. 1. For this work, detections are defined only by 2D bounding box in the image plane as presented in Fig. 1.

\subsection{Experimental Setting}

The assignment information are based on the distance between objects in the image plane. For that, the distance $d_{i, j}$ is defined as follows:

$$
d_{i, j}=\frac{1}{2}\left(d_{i, j}^{\text {right }}+d_{i, j}^{l e f t}\right),
$$

where $d_{i, j}^{\text {right }}$ (resp. $d_{i, j}^{\text {left }}$ ) is the Euclidean distance between bottom-right (resp. top-left) corners of the bounding boxes of target $X_{i}$ (detected object) and track $Y_{j}$ (known object) as presented in Fig. 2.

\footnotetext{
${ }^{3}$ http://www.cvlibs.net/datasets/kitti/eval_tracking.php.
} 


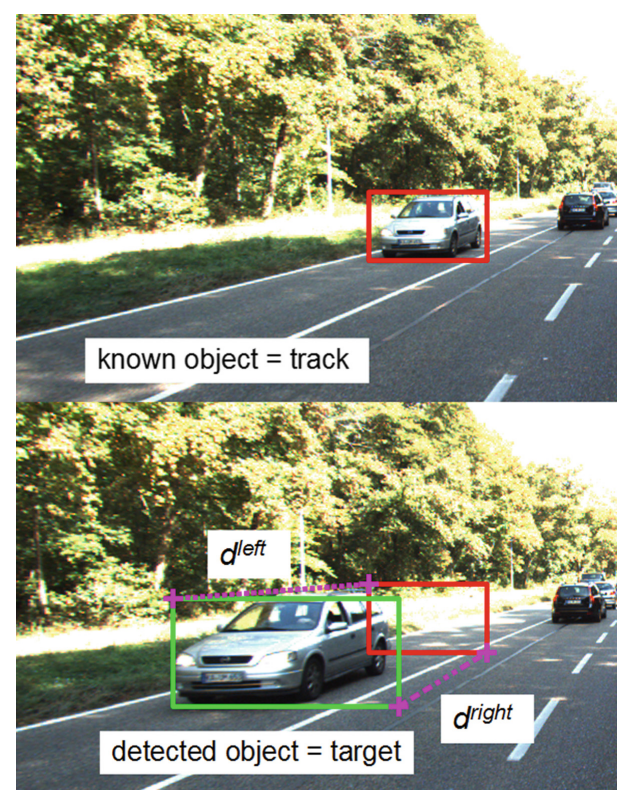

Fig. 2. The illustration of the distances $d_{i, j}^{\text {right }}$ and $d_{i, j}^{\text {left }}[4]$.

The parameters of the $b b a$ model (11) are: $\alpha=0.9$ and $\tau=0.5$. The index of similarity is defined as follows:

$$
I_{i, j}=\left\{\begin{aligned}
1-\frac{d_{i, j}}{D} & , \text { if } d_{i, j}<D \\
0 & , \text { otherwise },
\end{aligned}\right.
$$

where $D$ is the limit distance for association which is determined heuristically, e.g. $D=210$ in this work.

The tuning parameters $\epsilon=0.001$ and $q=5$ for $D S m P$ and MulP transformations respectively. The LPP algorithm has been used as optimization strategy in the decision-making step.

\subsection{Comparison of Probabilistic Transformations}

All discussed transformations are characterized by an equivalent complexity except the pignistic transformation. BetP is computed directly from combined masses which leads to a lower computational time.

To compare the performance of the probabilistic transformations presented previously, the object association system is evaluated by the True Associations Rate $(T A R)$ :

$$
T A R=\frac{\sum_{t} \text { True Association }_{t}}{\sum_{t} \text { Ground Truth }_{t}},
$$

where $t$ is the frame index. 
Table 2 compares association outcomes of the system based on different probabilistic transformations. Only target-to-track association results have been presented in Table 2 due to the lack of space. However, from track-to-target association results, similar comments/conclusions hold. The penultimate row of Table 2 shows the weighted average of TAR value based on all sequences which is given by:

$$
T A R_{\text {avg }}=\sum_{i=0}^{20} w_{i} T A R_{i}
$$

where $T A R_{i}$ is the TAR value of the $i$-th sequence, and where the weight $w_{i}$ is $w_{i}=n_{i} / \sum_{i=0}^{20} n_{i}$ and $n_{i}$ being the number of associations of the $i$-th sequence. For instance, $T A R_{\text {avg }}=0.9852$ (or $98.52 \%$ ) for the BetP transformation, etc. The last row of Table 2 represents the weighted standard deviation $\left(\sigma_{w}\right)$ of association scores defined as follows:

$$
\sigma_{w}=\sqrt{\sum_{n=0}^{20} w_{i}\left(T A R_{i}-T A R_{a v g}\right)^{2}}
$$

Table 2. target-to-track associations score (in \%) obtained by different probabilistic transformations.

\begin{tabular}{l|c|c|c|c|c|c|c|c|c|c}
\hline Seq. $n^{\circ}$ & nb of Frame & nb of Ass. & BetP & DSmP & PrPl & PrBel & MulP & $\operatorname{Pr} B P 1$ & $\operatorname{Pr} B \operatorname{P} 2$ & $\operatorname{Pr} B P 3$ \\
\hline Seq. 0 & 154 & 675 & $\mathbf{9 9 . 4 1}$ & 99.11 & $\mathbf{9 9 . 4 1}$ & 99.26 & $\mathbf{9 9 . 4 1}$ & $\mathbf{9 9 . 4 1}$ & 99.26 & 99.11 \\
\hline Seq. 1 & 447 & 2643 & $\mathbf{9 7 . 5 0}$ & 96.71 & 97.43 & 96.03 & 97.47 & 95.88 & 95.50 & 97.39 \\
\hline Seq. 2 & 233 & 668 & $\mathbf{9 9 . 7 0}$ & $\mathbf{9 9 . 7 0}$ & $\mathbf{9 9 . 7 0}$ & 97.75 & $\mathbf{9 9 . 7 0}$ & 98.65 & 97.46 & $\mathbf{9 9 . 7 0}$ \\
\hline Seq. 3 & 144 & 337 & $\mathbf{9 9 . 4 1}$ & $\mathbf{9 9 . 4 1}$ & $\mathbf{9 9 . 4 1}$ & 98.81 & $\mathbf{9 9 . 4 1}$ & 99.11 & 98.81 & $\mathbf{9 9 . 4 1}$ \\
\hline Seq. 4 & 314 & 545 & 89.72 & 93.39 & 93.21 & 92.29 & 90.09 & 92.29 & 91.56 & $\mathbf{9 3 . 7 6}$ \\
\hline Seq. 5 & 297 & 925 & 98.59 & 97.51 & 98.16 & 96.00 & $\mathbf{9 9 . 4 6}$ & 96.32 & 95.24 & 97.95 \\
\hline Seq. 6 & 270 & 474 & $\mathbf{1 0 0}$ & $\mathbf{1 0 0}$ & $\mathbf{1 0 0}$ & 98.95 & $\mathbf{1 0 0}$ & 98.95 & 98.73 & $\mathbf{1 0 0}$ \\
\hline Seq. 7 & 800 & 2084 & $\mathbf{9 7 . 6 0}$ & 96.55 & 97.17 & 95.11 & 97.55 & 95.25 & 94.63 & 97.02 \\
\hline Seq. 8 & 390 & 492 & 99.19 & 98.78 & 99.19 & 97.76 & $\mathbf{9 9 . 3 9}$ & 97.76 & 97.56 & 99.19 \\
\hline Seq. 9 & 802 & 2888 & $\mathbf{9 8 . 4 4}$ & 97.33 & 98.10 & 97.09 & 98.37 & 97.13 & 96.92 & 97.82 \\
\hline Seq. 10 & 294 & 541 & 98.71 & 98.34 & 98.71 & 97.78 & $\mathbf{9 9 . 2 6}$ & 98.15 & 98.89 & 98.34 \\
\hline Seq. 11 & 373 & 3001 & $\mathbf{9 9 . 3 7}$ & 98.77 & 99.30 & 99.30 & 99.33 & 99.27 & 99.27 & 99.23 \\
\hline Seq. 12 & 78 & 67 & $\mathbf{1 0 0}$ & $\mathbf{1 0 0}$ & $\mathbf{1 0 0}$ & $\mathbf{1 0 0}$ & $\mathbf{1 0 0}$ & $\mathbf{1 0 0}$ & $\mathbf{1 0 0}$ & $\mathbf{1 0 0}$ \\
\hline Seq. 13 & 340 & 617 & 93.35 & $\mathbf{9 5 . 6 2}$ & 94.00 & 93.35 & 93.19 & 93.35 & 92.06 & 94.00 \\
\hline Seq. 14 & 106 & 374 & 89.04 & 89.57 & 88.50 & 88.77 & 88.50 & $\mathbf{8 9 . 8 4}$ & 89.04 & 88.77 \\
\hline Seq. 15 & 376 & 1249 & $\mathbf{9 9 . 2 8}$ & $\mathbf{9 9 . 2 8}$ & $\mathbf{9 9 . 2 8}$ & 99.04 & $\mathbf{9 9 . 2 8}$ & 98.80 & 98.80 & $\mathbf{9 9 . 2 8}$ \\
\hline Seq. 16 & 209 & 1872 & $\mathbf{9 7 . 5 4}$ & 96.63 & 97.44 & 96.69 & $\mathbf{9 7 . 5 4}$ & 96.85 & 96.90 & 97.38 \\
\hline Seq. 17 & 145 & 486 & $\mathbf{9 9 . 1 8}$ & 98.35 & $\mathbf{9 9 . 1 8}$ & 96.71 & $\mathbf{9 9 . 1 8}$ & 97.33 & 96.91 & $\mathbf{9 9 . 1 8}$ \\
\hline Seq. 18 & 339 & 1130 & $\mathbf{9 9 . 8 2}$ & 98.41 & 99.65 & 98.94 & $\mathbf{9 9 . 8 2}$ & 99.03 & 98.94 & 99.29 \\
\hline Seq. 19 & 1059 & 4968 & $\mathbf{9 9 . 4 2}$ & 98.73 & $\mathbf{9 9 . 4 2}$ & 97.83 & 99.36 & 97.89 & 97.36 & 99.34 \\
\hline Seq. 20 & 837 & 4673 & $\mathbf{9 9 . 6 8}$ & 98.35 & 99.59 & 98.35 & 99.64 & 98.20 & 98.10 & 99.42 \\
\hline All Seq. & 8007 & 30709 & $\mathbf{9 8 . 5 2}$ & 97.85 & $\mathbf{9 8 . 4 7}$ & 97.35 & $\mathbf{9 8 . 5 2}$ & 97.40 & 97.10 & 98.37 \\
\hline & std. dev. & $\sigma w$ & 1.38 & $\mathbf{1 . 0 5}$ & 1.22 & 1.26 & 1.39 & 1.21 & 1.36 & 1.18 \\
\hline & & & & & & & & & &
\end{tabular}


The obtained results show that $\operatorname{Pr} B e l, \operatorname{Pr} B P 1$, and $\operatorname{Pr} B P 2$ provide the worst mean associations scores $(\leq 97.40 \%)$ with the largest standard deviation $(1.36 \%)$ for $\operatorname{Pr} B P 2$. It can be explained by the fact that these transformations are based on the $\mathrm{Bel}$ function which is a pessimistic measurement. The rest of the transformations provide rates of correct association (i.e. scores) $>98.40 \%$ which represents a gain of $+1 \%$. The best mean score $\approx 98.50 \%$ is given by $B e t P, P r P l$, and $M$ ult $P$ transformations. Based only on the mean score criterion, Bet $P$ seems more interesting because it provides better scores on 15 sequences from 21 as illustrated in Fig. 3. In addition, Bet $P$ is based on a very simple transferring process of uncertainty which makes $\operatorname{Bet} P$ a good choice for real-time applications. However, this apparent advantage of $\operatorname{Bet} P$ needs to be seen in relative terms because $\operatorname{Bet} P$ also generates a quite large standard deviation of $1.38 \%$, which clearly indicate that $B$ et $P$ is not very precise. $P r P l$ and $M$ ult $P$ are also characterized by a relatively high standard deviation $(1.22 \%$ and $1.39 \%)$. On the other hand, the lower standard deviation $1.05 \%$ is given by $D S m P$ transformation with a good association score $=97.85 \%$. This transformation performs well in term of PCI criteria which leads to make correct decisions [11]. Consequently, $D S m P$ is an interesting alternative to BetP for the data association process in autonomous vehicle perception system.

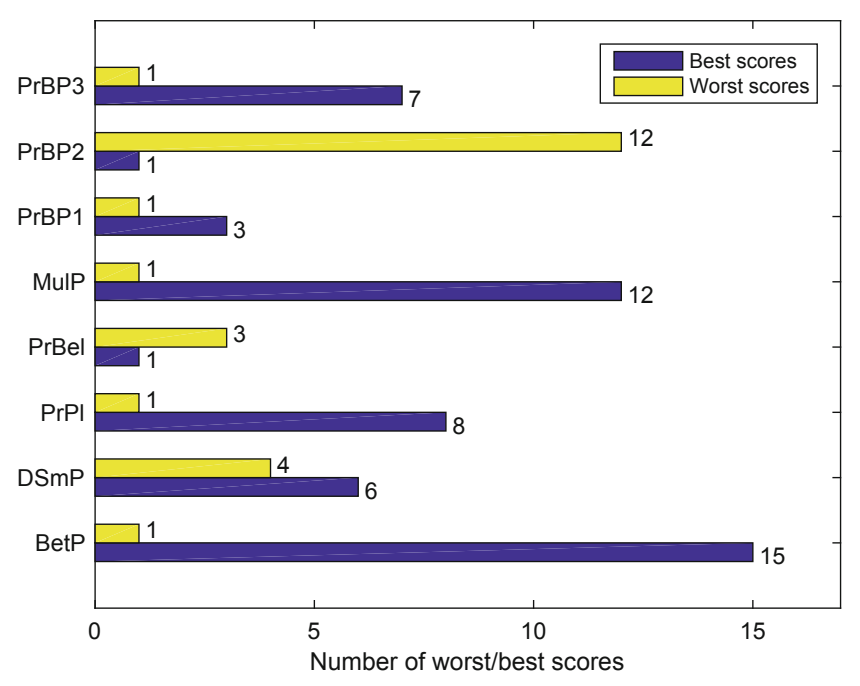

Fig. 3. The number of worst/best scores obtained by each probabilistic transformation on 21 sequences; e.g. $\operatorname{PrBel}$ provides three worst scores (sequences 3, 10, and 17) and only one best score on sequence 12 . 


\section{Conclusion}

An evaluation of several probabilistic transformations for evidential data association has been presented in this paper. These transformations approximate the belief masses by a probability measure in order to make association decisions. The widely used probabilistic approximation is the pignistic transformation. However, several published studies criticize the choice of this method of approximation and propose generalized transformations.

We did compare the performances of these probabilistic transformations on real-data in order to determine which one is more suited for assignment problems in the context of autonomous vehicle navigation based on real datasets. The obtained results based on the well-known KITTI dataset show that the pignistic transformation provides one of the better scores. However, it provides a quite large standard deviation contrary to $D S m P$ transformation which provides the lowest standard deviation. In addition, $D S m P$ procures a nearly similar association score to that given by $\operatorname{BetP}$. Consequently, $D S m P$ can be a good alternative to $B e t P$ for the autonomous vehicle perception problematic requiring a bit more computational power with respect to BetP.

\section{References}

1. Bar-Shalom, Y., Li, X.: Multitarget-Multisensor Tracking: Principles and Techniques. YBS Publishing, Brandford (1995)

2. Blackman, S., Popoli, R.: Design and Analysis of modern Tracking Systems. Artech House, Norwood (1999)

3. Blackman, S.: Multiple-target Tracking with Radar Applications. Artech House Radar Library, Norwood (1986)

4. Boumediene, M.: Evidential data association: benchmark of belief assignment models. In: International Conference on Advanced Electrical Engineering, Algeria, November 2019. https://doi.org/10.1109/ICAEE47123.2019.9014699

5. Boumediene, M., Lauffenburger, J.P., Daniel, J., Cudel, C.: Coupled detection, association and tracking for traffic sign recognition. In: IEEE Intelligent Vehicle Symposium, Dearborn, Michigan, USA, June 2014, pp. 1402-1407. https://doi. org/10.1109/IVS.2014.6856492

6. Boumediene, M., Lauffenburger, J.P., Daniel, J., Cudel, C., Ouamri, A.: Multi-ROI association and tracking with belief functions: application to traffic sign recognition. IEEE Trans. Intell. Trans. Syst. 15(6), 2470-2479 (2014). https://doi.org/10. 1109/TITS.2014.2320536

7. Cobb, B., Shenoy, P.: On the plausibility transformation method for translating belief function models to probability models. IJAR 41(3), 314-330 (2006)

8. Cuzzolin, F.: On the properties of the intersection probability, submitted to the Annals of Mathematics and AI (2007)

9. Dempster, A.P.: Upper and lower probabilities induced by a multiple valued mapping. Ann. Math. Statistics 38, 325-339 (1967)

10. Denœux, T., El Zoghby, N., Cherfaoui, V., Jouglet, A.: Optimal object association in the dempster-shafer framework. IEEE Trans. Cybern. 44(22), 2521-2531 (2014). https://doi.org/10.1109/TCYB.2014.2309632 
11. Dezert, J., Smarandache, F.: A new probabilistic transformation of belief mass assignment. In: International Conference on Information Fusion (FUSION), Cologne, Germany , pp. 1410-1417. (2008)

12. Fortmann, T.E., Bar-Shalom, Y., Scheffe, M.: Sonar tracking of multiple targets using joint probabilistic data association. IEEE J. Oceanic Eng. 8(3), 173-184 (1983). https://doi.org/10.1109/JOE.1983.1145560

13. Geiger, A., Lenz, P., Urtasun, R.: Are we ready for autonomous driving? The KITTI vision benchmark suite. In: Conference on Computer Vision and Pattern Recognition (CVPR), Rhode Island, USA (2012). https://doi.org/10.1109/CVPR. 2012.6248074

14. Gruyer, D., Demmel, S., Magnier, V., Belaroussi, R.: Multi-hypotheses tracking using the Dempster-Shafer theory, application to ambiguous road context. Inf. Fusion 29, 40-56 (2016)

15. Gruyer, D., Berge-Cherfaoui, V.: Multi-objects association in perception of dynamical situation. In: Proceedings of the Fifteenth Conference on Uncertainty in Artificial Intelligence, UAI 1999, pp. 255-262. Morgan Kaufmann Publishers Inc., San Francisco (1999)

16. Hachour, S., Delmotte, F., Mercier, D.: Comparison of credal assignment algorithms in kinematic data tracking context. In: Laurent, A., Strauss, O., BouchonMeunier, B., Yager, R.R. (eds.) IPMU 2014. CCIS, vol. 444, pp. 200-211. Springer, Cham (2014). https://doi.org/10.1007/978-3-319-08852-5_21

17. Lauffenburger, J.P., Boumediene, M.: Adaptive credal multi-target assignment for conflict resolving. In: International Conference on Information Fusion (FUSION), Heidelberg, Germany, July 2016, pp. 1578-1584

18. Lauffenburger, J.P., Daniel, J., Boumediene, M.: Traffic sign recognition: benchmark of credal object association algorithms. In: International Conference on Information Fusion (FUSION), Salamanca, Spain, July 2014, pp. 1-7

19. Li, M., Lu, X., Zhang, Q., Deng, Y.: Multiscale probability transformation of basic probability assignment. Math. Prob. Eng. 2014, 6 (2014)

20. Mercier, D., Lefèvre, E., Jolly, D.: Object association with belief functions, an application with vehicles. Inf. Sci. 181(24), 5485-5500 (2011). https://doi.org/10. 1016/j.ins.2011.07.045

21. Pan, W., Yang, H.: New methods of transforming belief functions to pignistic probability functions in evidence theory. In: International Workshop on Intelligent Systems and Applications, China, May 2009

22. Reid, D.: An algorithm for tracking multiple targets. IEEE Trans. Autom. Control 24(6), 843-854 (1979). https://doi.org/10.1109/TAC.1979.1102177

23. Rombaut, M.: Decision in multi-obstacle matching process using Dempster-Shafer's theory. In: International Conference on Advances in Vehicle Control and Safety, Amiens, France, pp. 63-68 (1998)

24. Royère, C., Gruyer, D., Cherfaoui, V.: Data association with believe theory. In: International Conference on Information Fusion (FUSION), Paris, France, July 2000. https://doi.org/10.1109/IFIC.2000.862698

25. Shafer, G.: A Mathematical Theory of Evidence. Princeton University Press, Princeton (1976)

26. Smets, P.: The combination of evidence in the transferable belief model. IEEE Trans. Pattern Anal. Mach. Intell. 12, 447-458 (1990)

27. Smets, P., Kennes, R.: The transferable belief model. Artif. Intell. 66(2), 191-234 (1994). https://doi.org/10.1016/0004-3702(94)90026-4

28. Smets, P.: Decision making in the tbm: the necessity of the pignistic transformation. Int. J. Approximate Reasoning 38, 133-147 (2005) 
29. Smets, P.: Analyzing the combination of conflicting belief functions. Inf. Fusion 8(4), 387-412 (2007). https://doi.org/10.1016/j.inffus.2006.04.003

30. Sudano, J.: Pignistic probability transforms for mixes of low- and high-probability events. In: International Conference on Information Fusion, Montreal, August 2001

31. Sudano, J.: The system probability information content (pic) relationship to contributing components, combining independent multi-source beliefs, hybrid and pedigree pignistic probabilities. In: International Conference on Information Fusion, Annapolis, July 2002 